Año 13.

Revista de Investigación

Núm. 33

Académica sin Frontera

ISSN: 2007-8870

http://revistainvestigacionacademicasinfrontera.com

Recibido el 15 de octubre de 2020. Dictaminado mediante arbitraje favorablemente 20 de diciembre de 2020.

\title{
Comparando protocolos centralizados vs distribuídos para redes inalámbricas de sensores.
}

\section{Comparing centralized vs distributed protocols for wireless sensor networks.}

\author{
Dr. Joel Ruiz Ibarra ${ }^{1}$ \\ Mtro. Edgar Espinoza Zallas ${ }^{2}$ \\ Mtra. Patricia Andrade Salinas ${ }^{3}$ \\ Mtro. Juan Pablo Aguilar Limón ${ }^{4}$ \\ Dra. Erica Ruiz Ibarra ${ }^{5}$
}

Resumen:

La academia, la industria y la sociedad son algunas de las áreas de aplicación del Internet de las Cosas, creando nuevos servicios. Sin embargo, el análisis del consumo de energía de los componentes de la red, en partocupar durante el establecimiento de rutas, es un tema de gran interés debido a varios estudios y simulaciones. Las API que se proponen en este trabajo permiten evaluar protocolos de enrutamiento y aplicaciones por medio de interfaces sencillas, desarrolladas en C\#, mejorando el desarrollo de aplicaciones IoT. Se emplea una red de sensores implementada con módulos XBee para evaluar la propuesta en un escelario real. Los resultados muestran que las API propuestas obtienen desempeños positivos muy similares implementadas y simuladas.

\footnotetext{
${ }^{1}$ https://orcid.org/0000-0002-4932-2006

${ }^{2}$ https://orcid.org/0000-0001-7667-5782

${ }^{3}$ https://orcid.org/0000-0002-9723-3964

${ }^{4}$ https://orcid.org/0000-0003-1811-9803

${ }^{5}$ https://orcid.org/0000-0002-7020-4960
} 
Año 13.

Revista de Investigación

Núm. 33

Académica sin Frontera

ISSN: 2007-8870

\title{
http://revistainvestigacionacademicasinfrontera.com
}

Recibido el 15 de octubre de 2020. Dictaminado mediante arbitraje favorablemente 20 de diciembre de 2020.

Palabras Claves: IoT, AODV, WSN, QUATTRO, Energía

\begin{abstract}
Academy, industry, and society are finding many areas of application of the Internet of Things, creating new services. However, the analysis of energy consumption carried out by the components of the network, in specific during the establishment of the routing, is a hot topic because many studies are carried out by simulations. The proposed API permits evaluate routing protocols and applications by means of a friendly interface developed using C\# introducing development improvement to a wide set of IoT applications. Moreover, a wireless sensor was created using XBee Series 1 and IEEE 802.15.4 standard to evaluate protocols and applications through a real scenario. The energy consumption analysis carried out with the API and wireless sensor is very similar with the results obtained by simulation.
\end{abstract}

Keywords: IoT, AODV, WSN, QUATTRO, Energy.

\section{INTRODUCCIÓN}

El Internet de las Cosas (IoT) se define como: infraestructura de red global dinámica con la capacidad de auto-configurarse, basada en estándares y protocolos de comunicación interoperables donde cosas físicas y virtuales, con identidad y atributos, utilizan interfaces integradas dentro de la red de información. El término de Internet de las cosas o Internet of Things, se le atribuye a Kevin Ashton y a David L. Brock (Pascual, 2012), haciendo referencia a la posibilidad de que cualquier objeto o cosa pueda realizar una comunicación. La comunicación se puede realizar a través de cualquier tecnología, ya sea alámbrica o inalámbrica. 


\section{Año 13.}

Revista de Investigación

Núm. 33

Académica sin Frontera

ISSN: 2007-8870

\section{http://revistainvestigacionacademicasinfrontera.com}

Recibido el 15 de octubre de 2020. Dictaminado mediante arbitraje favorablemente 20 de diciembre de 2020.

Hace pocos años, el concepto de IoT se percibía como una visión futurista; sin embargo, el mercado sobre el uso y aplicación de la tecnología va en aumento, permitiendo incrementar la productividad en las empresas e incrementando la posibilidad de tecnologías disruptivas (Zorzy et. al 2010, Chen et. al. 2014).

La posibilidad comercial del IoT es amplia y diversa, ya que la podemos encontrar o aplicar en los sectores de transporte, energía, educación, logística, e industrial (Zorzy et. al 2010, Chen et. al. 2014). En este sentido, se ha detectado como oportunidad de negocio al Internet de las Cosas en el hogar, específicamente en administrar, monitorear y surtir gas L.P. en los hogares y negocios (Vermesan et. al. 2014, Hung 2017). El alcance del IoT va más allá de la comunicación máquina a máquina (M2M, por sus siglas en inglés Machine to Machine) por ser robusto en protocolos, aplicaciones y dominios de red (Paul 2017). Es un entorno total que interactúa con nuestras actividades cotidianas para brindarnos confort en relación a nuestras necesidades.

En esta propuesta se desarrolla una serie de funciones a modo de una Interfaz de Programación de Aplicaciones (API) que permita introducir al concepto IoT a una amplia gama de objetos y aplicaciones. Además, se ha generado un modelo de consumo de energía para evaluar el gasto de energía que requieren algoritmos (Zhang et. al. 2009). Para demostrar su eficiencia, se compara el desempeño de dos algoritmos de enrutamiento, AODV (Perkin 2000) y QUATTRO (Ruiz 2009).

En la sección 2, se describen los trabajos relacionados. En la sección 3, se explica el desarrollo de la propuesta que consiste en el desarrollo de la API y el sensor inalámbrico. 


\section{Año 13.}

Revista de Investigación

Núm. 33

Académica sin Frontera

ISSN: 2007-8870

\section{http://revistainvestigacionacademicasinfrontera.com}

Recibido el 15 de octubre de 2020. Dictaminado mediante arbitraje favorablemente 20 de diciembre de 2020.

\section{TRABAJOS RELACIONADOS}

Los protocolos de enrutamiento bajo demanda (o en inglés on-demand routing protocols) tienen la característica de enviar paquetes para crear una ruta cuando un nodo requiere enviar datos a un destino, evitando enviar paquetes de manera constante para mantener una tabla de enrutamiento (Srivastava et. al. 2014). La base de cualquier protocolo bajo demanda es el DSR (Dynamic Source Routing) (Johnson y Maltz 1996) que gestiona las rutas entre nodos que participan en la comunicación, reduciendo la carga del procesador y consumo de energía. Un protocolo que mejora las prestaciones de DSR es AODV (Ad-hoc On-demand Distance Vector) a través de mantener tablas de enrutamiento en cada nodo participante, evitando adjuntar información de la ruta en cada paquete (Perking et. al. 2000). A partir de estos protocolos de enrutamiento, se han propuesto otras alternativas (Giannoulis et. al. 2007, Jiang et. al. 1999) que han sido evaluados en términos de consumo de energía (Dengler et. al. 2007, Anastasi et. al. 2009, Fotino et. al. 2007, Fall y Varadhan 2000, Sultan y Zaki 2017, Kanakaris et. al. 2010) Otros trabajos han comparado los protocolos en escenarios reales (Nadilma, C.V.N Pereira y Renato M. de Moraes, 2010) y (Ferronato, J.J. y Trentin, M.A.S, 2017).

En Sultan, T. M. y Zaki, S. M, 2017 se analizan los protocolos AODV, DSR y OLSR para conocer el consumo de energía con respecto al crecimiento de los nodos, demostrando que el protocolo AODV consume más energía al inicio del protocolo que DSR y OLSR. En Kanakaris et. al. 2010 se analizan los protocolos DSR, AODV, DSDV, y TORA con el simulador NS-2. La simulación considera hasta 30 nodos móviles a una velocidad de $20 \mathrm{~m} / \mathrm{s}$ y con una energía inicial de 1000 Joules donde no se encontró diferencia significativa en el consumo de energía entre los cuatro protocolos evaluados; sin embargo, cuando el número de nodos se incrementa, el consumo de energía del protocolo TORA es ineficiente. 
Año 13.

Revista de Investigación

Núm. 33

Académica sin Frontera

ISSN: 2007-8870

\section{http://revistainvestigacionacademicasinfrontera.com}

Recibido el 15 de octubre de 2020. Dictaminado mediante arbitraje favorablemente 20 de diciembre de 2020.

\section{MARCO DE TRABAJO.}

\section{A. Módulo Sensor Inalámbrico}

Se utiliza un transmisor/receptor XBee Serie 1 que provee de conectividad inalámbrica entre dispositivos de la misma familia a precios razonables comparado con otras opciones como Freescale o Crossbow. XBee emplea dos modos de operación, uno en el que los datos que recibe en su puesto en el puerto serial (modo AT) y otro modo en el que se puede controlar el flujo de la transmisión, conocer potencia de señal recibida (RSSI), definir potencia de transmisión y dirección de destino entre otros parámetros (Modo API). Se diseñó un módulo transmisor/receptor que incluye un XBee S1 y un PIC16F4550 como se muestra en la Figura 1.

Figura 1. Nodo WSN, LCD con PIC16F4550

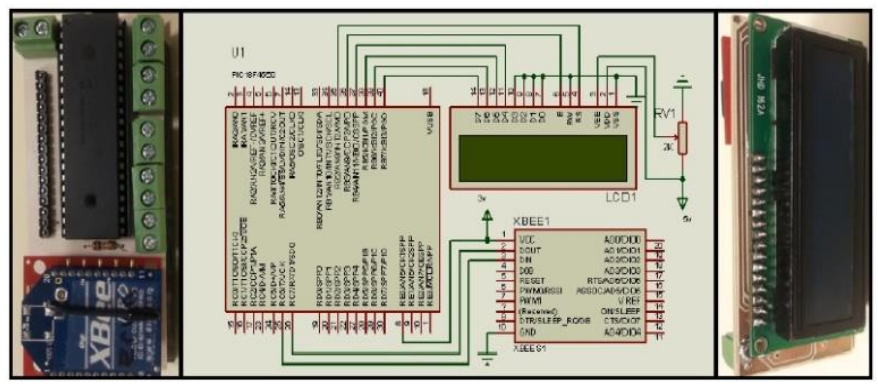

A partir del módulo, se desarrollan funciones de comunicaciones que facilitan la interacción con el módulo transmisor XBee S1 y sus diferentes parámetros de configuración y operación. Se busca reducir el tiempo de desarrollo de aplicaciones y propicia la evaluación aplicada de protocolos de comunicaciones para redes ad-hoc. Se utiliza el modo API para acceder a la configuración y control de flujo de datos del módulo XBee. La trama XBee original se muestra en la Figura 2 donde el campo específico de estructura API recibe las 
Año 13.

Revista de Investigación

Académica sin Frontera

Núm. 33

ISSN: 2007-8870

\section{http://revistainvestigacionacademicasinfrontera.com}

Recibido el 15 de octubre de 2020. Dictaminado mediante arbitraje favorablemente 20 de diciembre de 2020.

instrucciones de programación de aplicación que aquí se proponen para mejorar el desempeño de una WSN aplicada a proveer interconectividad a objetos que originalmente eran pasivos, volviéndose parte del IoT.

Figura 2. Trama API del módulo XBee

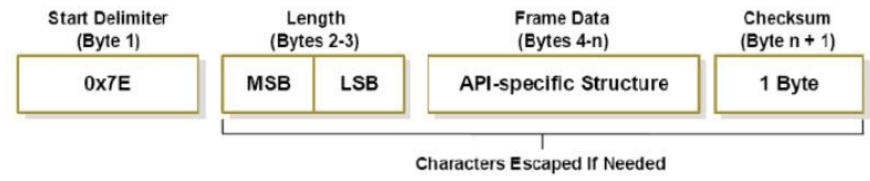

Tanto la información que va destinada a comunicarse por radio frecuencia como las instrucciones locales y confirmaciones de recibido para el módulo XBee, emplean el formato de trama de la Figura 2.

Cada vez que se inicia una comunicación con WSN, se intercambia información de configuración y de reconocimiento de vecinos para formar la topología de una red ad-hoc. Este intercambio de paquetes debe darse en un mismo entorno de red, definido por el identificador de red de área personal (PANID) y un número de identificación único para cada nodo en la red (NODEID). El direccionamiento presente en una WSN basada en el estándar IEEE 802.15.4 permite direccionar más de 65500 nodos, sin embargo en la práctica hemos visto que ese número se reduce drásticamente, dependiendo del protocolo de coordinación y enrutamiento. 
"El saber de mis hijos

Año 13.

Núm. 33
Revista de Investigación

Académica sin Frontera

ISSN: 2007-8870

\section{http://revistainvestigacionacademicasinfrontera.com}

Recibido el 15 de octubre de 2020. Dictaminado mediante arbitraje favorablemente 20 de diciembre de 2020.

\section{B. Propuesta de Interfaz API}

Esta propuesta utiliza C\# para desarrollar una interfaz de programación entre la aplicación y la Red Inalámbrica de Sensores (WSN) real implementada. Para ello se desarrolló una clase, nombrada XbeeBaseFrame que se muestra en la Figura 3.

Figura 3. Clase XBeeBaseFrame

\begin{tabular}{|c|c|}
\hline $\begin{array}{l}\text { XbeebaseFrame } \\
\text { Class }\end{array}$ & 同 \\
\hline \multicolumn{2}{|l|}{$\boxminus$ Fields } \\
\hline $\begin{array}{l}\text { checksum } \\
\text { fatalen } \\
\\
\text { flag } \\
\text { Txhist }\end{array}$ & \\
\hline \multicolumn{2}{|l|}{ G Properties } \\
\hline $\begin{array}{l}\text { CheckSum } \\
\text { DataLen } \\
\text { Payload } \\
\text { Port }\end{array}$ & \\
\hline \multicolumn{2}{|l|}{$\Xi$ Methods } \\
\hline $\begin{array}{l}=\text { BuiltFrame } \\
=\text { DisplayFrame } \\
=\text { SendFrame } \\
=\text { XbeeBaseFrame (+ } 8 \text { overloads) }\end{array}$ & \\
\hline \multicolumn{2}{|l|}{$\because$ Nested Types } \\
\hline $\begin{array}{l}\text { Flags } \\
\text { Enum }\end{array}$ & $\approx$ \\
\hline
\end{tabular}

La interfaz API WSN que aquí se propone consiste en un conjunto de clases como la mostrada en la Figura 3 que facilita el acceso a los recursos de configuración y comunicación sobre el estándar IEEE 802.15.4. Esta propuesta se basa en un módulo de comunicación punto 


\section{Año 13.}

Revista de Investigación

Núm. 33

\section{http://revistainvestigacionacademicasinfrontera.com}

Recibido el 15 de octubre de 2020. Dictaminado mediante arbitraje favorablemente 20 de diciembre de 2020.

a punto XBee S1, logrando extender sus capacidades hasta formar una red WSN Ad-Hoc auto-configurable y enrutable.

La clase XBeeBaseFrame contiene campos públicos y privados que tienen tal robustez que sólo pueden ser modificados de la manera correcta para que el módulo XBee pueda interpretarlos. Inicia una trama de datos con un byte delimitador $(0 x 7 \mathrm{E})$ y finaliza con un byte de detección de errores (Checksum). Si bien la comunicación puede cifrarse para ofrecer cierto grado de seguridad, esta propuesta se limita a mejorar la curva de desarrollo de aplicaciones, dejando como trabajo subsecuente el proveer de mecanismos apropiados de seguridad en función de la aplicación y carga de procesamiento requerido. Gracias a esta clase, el usuario es capaz de construir tramas de datos correctamente en un paquete API, interpretable por el módulo XBee. La Figura 4 muestra el método constructor de dicha trama. Basta con la carga útil y el puerto de comunicaciones a emplear para que esta función construya un paquete API con su byte delimitador de inicio, identificador de paquete, carga útil, dirección de destino y byte detector de errores.

Figura 4. Constructor de trama API para XBee S1

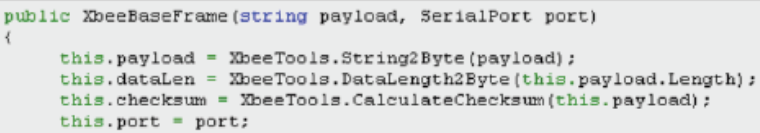




\section{Año 13.}

Revista de Investigación

Núm. 33

\section{http://revistainvestigacionacademicasinfrontera.com}

Recibido el 15 de octubre de 2020. Dictaminado mediante arbitraje favorablemente 20 de diciembre de 2020.

AODV es uno de los protocolos más utilizados para redes Ad-Hoc por ser reactivo y de fácil implementación. En esta propuesta se incluye el desarrollo de una clase que permite implementar el protocolo AODV en una WSN para tener referencia con la cual comparar nuevas implementaciones de enrutamiento. Esta clase incluye campos como 16 bits de dirección, ID de trama, ID de petición de ruta, número de secuencia entre otras propias de AODV para la configuración del canal de radiocomunicaciones. Los campos de la clase AODV se muestran en la Figura 5.

Figura5. Clase AODV

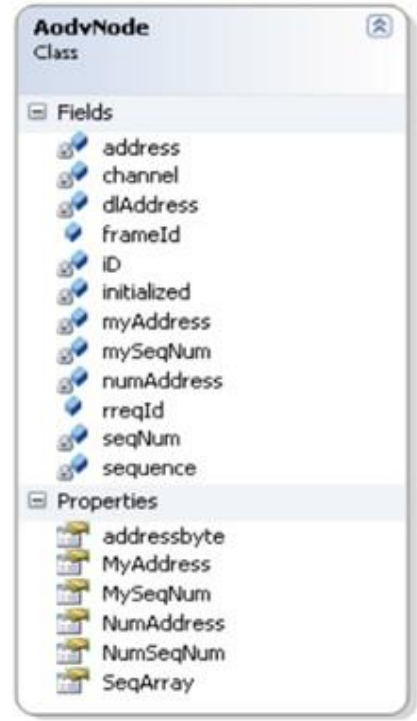

\section{Complejidad Computacional}

Como podemos ver en la Figura 6, esta propuesta no contiene ciclos recursivos o cálculos dependientes del número de nodos que escalen en la misma proporción que el tamaño de la red. El tiempo de ejecución de las funciones aquí propuestas es relativamente corto como se 
"El saber de mis hijos hará mi grandeza"

Año 13.
Revista de Investigación Académica sin Frontera ISSN: 2007-8870

\section{http://revistainvestigacionacademicasinfrontera.com}

Recibido el 15 de octubre de 2020. Dictaminado mediante arbitraje favorablemente 20 de diciembre de 2020. mostrará en el apartado de desempeño más adelante. Esto hace que esta interfaz API propuesta para WSN encaje en el orden de complejidad tipo polinomial. De este modo, el mayor orden de complejidad dependerá de los protocolos que se desarrollen sobre esta interfaz.

Figura 6. Diagrama de flujo para transmisión/recepción
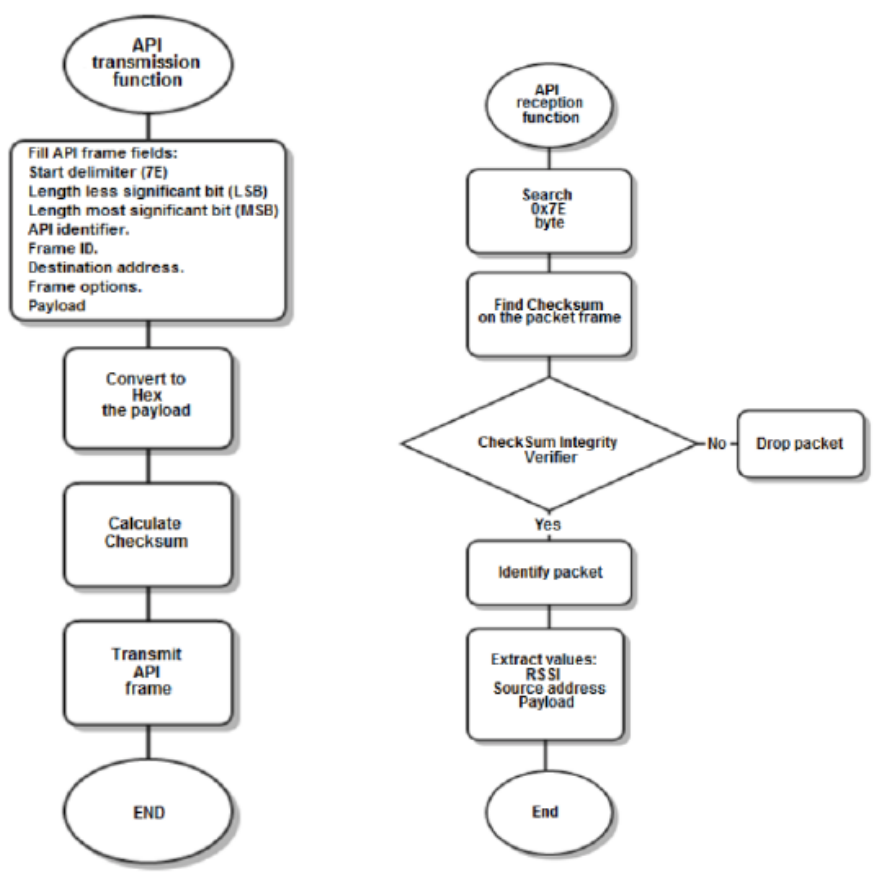

\section{Modelo de Consumo de Energía}

El consumo de energía en una WSN sigue un comportamiento exponencial negativo. Se modeló un escenario de 121 nodos distribuidos aleatoria y heterogéneamente en un espacio de 400x400m como se muestra en la Figura 7. 
Año 13.

Núm. 33

Académica sin Frontera

ISSN: 2007-8870

\section{http://revistainvestigacionacademicasinfrontera.com}

Recibido el 15 de octubre de 2020. Dictaminado mediante arbitraje favorablemente 20 de diciembre de 2020.

Figura 7. Escenario simulado

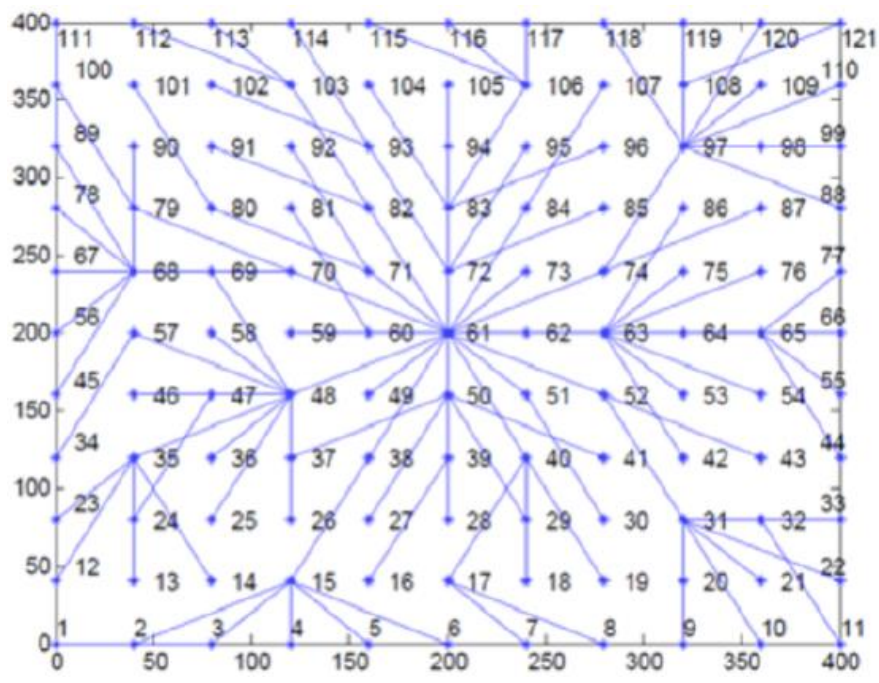

La Figura 7 muestra un esquema de enrutamiento iniciado por el nodo \#61, Sumidero (Sink). El Sink difunde su disponibilidad de ruta a los nodos a un salto en el tiempo de difusión 1. Aquellos nodos que lo escuchan ya conocen un camino hacia el Sink y difunden la disponibilidad de una ruta hacia él en el tiempo de difusión 2. Los nodos en el segundo salto al Sink escuchan esta disponibilidad de ruta y seleccionan a uno de los nodos fuente como ruta padre y al resto como rutas alternas. Se repite el proceso de difusión hasta que después de $\mathrm{n}$ difusiones la red completa conoce al menos una ruta al Sink, donde $\mathrm{n}$ es el número de saltos de la ruta más larga hacia el Sink. Este método de enrutamiento no es orientado a calidad de servicio (QoS) u orientado al ahorro de energía, el único propósito es encontrar una ruta al Sink y evaluar el modelo de consumo de energía.

La ecuación (1) muestra el voltaje estimado en la batería en función de la carga de tráfico del nodo. Esta ecuación es el resultado heurístico de mediciones de consumo de energía en la mesa de pruebas diseñada para esta propuesta. 
Año 13.

Revista de Investigación

Núm. 33

\section{http://revistainvestigacionacademicasinfrontera.com}

Recibido el 15 de octubre de 2020. Dictaminado mediante arbitraje favorablemente 20 de diciembre de 2020.

$$
V_{b a t t}=V_{o p}+\left(V_{\max }-V_{o p}\right) e^{-L / \beta}
$$

\section{Donde:}

$V_{\text {batt }}=$ Voltaje remanente en la batería .

$V_{o p}=$ Voltaje mínimo de operación

$V_{\max }=$ Nivel inicial de voltaje en la batería

$\beta=$ Factor de atenuación

$L=$ Carga de tráfico del nodo

Definición de tiempo de vida: El tiempo de vida de una red es el período que comprende desde que se despliega hasta el instante en que la red es considerada no funcional. Una red puede ser considerada no funcional en diferentes circunstancias, dependiendo de la aplicación. Por ejemplo, puede ser el instante en el que un primer nodo agote su energía (muere), o bien cuando un porcentaje de nodos mueren, o un segmento de la red quede desconectado del resto (W. Hu et. al. 2019) (Sara N et. al. 2017).

\section{DESEMPEÑO}

\section{A. Consumo de Energía, modelo simulado vs implementado.}

Para fines de evaluación, en esta propuesta se considera el tiempo de vida hasta que un primer nodo agote su energía. La Figura 7 muestra las rutas generadas con el esquema simplificado de enrutamiento para evaluar el consumo de energía correspondiente. La Figura 8 muestra el consumo de energía de ese mismo escenario. Como podemos ver, los nodos que estás cerca 


\section{Año 13.}

Revista de Investigación

Núm. 33

Académica sin Frontera

ISSN: 2007-8870

\section{http://revistainvestigacionacademicasinfrontera.com}

Recibido el 15 de octubre de 2020. Dictaminado mediante arbitraje favorablemente 20 de diciembre de 2020.

del Sink consumen más energía que aquellos que están más alejados debido a su carga de enrutamiento.

Tal como se midió en nodos reales, el voltaje máximo de su batería fue de $2.8 \mathrm{v}$ mientras que el voltaje mínimo de operación fue de $2.2 \mathrm{v}$. El consumo de energía no homogéneo que se observa es debido a que los nodos más cercanos al Sink deben retransmitir la información proveniente de los nodos más alejados, mientras que estos últimos sólo envían sus propios datos sin reenviar datos ajenos.

En la Figura 8 los nodos cercanos al Sink se quedan sin energía cuando los nodos de los extremos más alejados al Sink de la red aún tienen el 90\% de su batería.

Cada vez que un nodo Sink exista y capte la información de toda la red, se va a manifestar esta tendencia, agotando la energía principalmente de aquellos nodos cercanos al Sink. Se implementó un módulo sensor físico para medir el consumo de energía en ambientes reales. Este nodo consiste de un microcontrolador PIC 18F4550, un despliegue de cristal líquido (LCD), convertidor de corriente a voltaje y un módulo XBee S1. Es razonable pensar que el PIC consumirá la mayoría de la energía del nodo, más que el XBee. Dado que en esta propuesta se evalúan las tareas de comunicaciones, se utilizan fuentes diferentes para el PIC como para el módulo XBee. El PIC está alimentado por una fuente sin restricciones, mientras que el módulo XBee emplea un conjunto de dos baterías de 900mAh. La tarea realizada por el PIC puede ser realizada por una amplia gama de microcontroladores, por lo que su consumo varía en función del fabricante. Este modelo de consumo de energía analiza sólo el consumo debido a transmisión y recepción del módulo XBee, producto del algoritmo de comunicaciones. 
"El saber de mis hijos

Año 13.

Núm. 33
Revista de Investigación

Académica sin Frontera

ISSN: 2007-8870

\section{http://revistainvestigacionacademicasinfrontera.com}

Recibido el 15 de octubre de 2020. Dictaminado mediante arbitraje favorablemente 20 de diciembre de 2020.

Figura 8. Energía consumida en la simulación de red

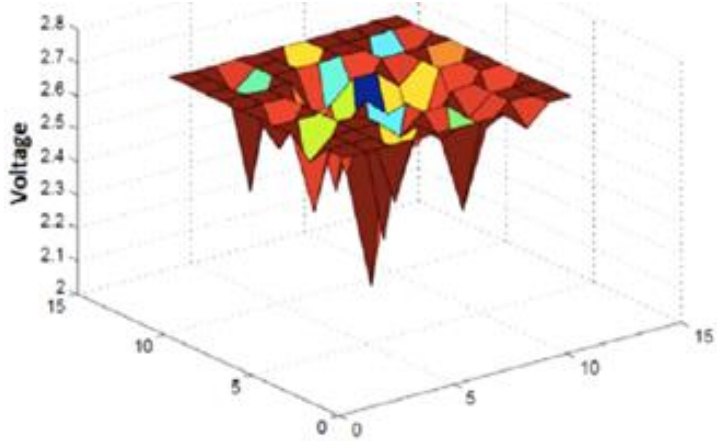

La Figura 9 muestra el circuito diseñado para medir el consumo de energía de un nodo a un bajo costo. Se miden el voltaje de la batería y la corriente del nodo. Utilizando un convertidor de corriente a voltaje se obtienen $1 \mathrm{mV}$ por cada $1 \mathrm{~mA}$ consumido. De este modo, multiplicando el voltaje de la batería por la conversión a voltaje de la corriente se obtiene la potencia instantánea consumida. Si se integra este consumo de potencia en un instante de tiempo, se obtiene la cantidad de energía consumida en ese tiempo.

Figura 9. Hardware para medir el consumo de energía

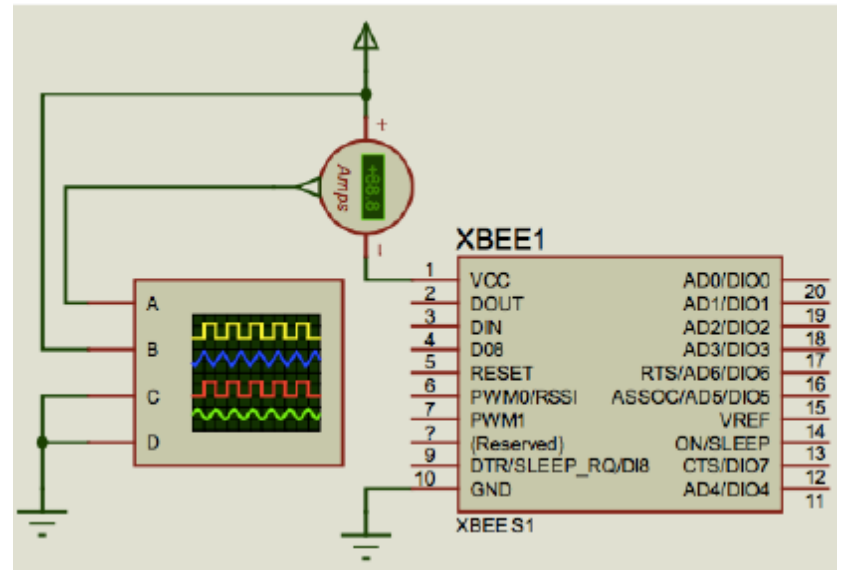




\section{Año 13.}

Revista de Investigación

Núm. 33

Académica sin Frontera

ISSN: 2007-8870

\section{http://revistainvestigacionacademicasinfrontera.com}

Recibido el 15 de octubre de 2020. Dictaminado mediante arbitraje favorablemente 20 de diciembre de 2020.

La temperatura es un factor importante al momento de considerar la descarga de una batería. Las mediciones de consumo de energía que aquí se presentan se obtuvieron en un ambiente de exteriores a una temperatura que varió de 27 a 31 grados Centígrados. Se transmiten paquetes de 100bytes utilizando el modo API entre un nodo A y un nodo B sin cambiar a modo dormir. Según las mediciones, el módulo XBee consume de 43mA a 48mA, agotando sus baterías como se muestra en la Figura 10. Esta configuración tomó diecinueve horas para agotar dos baterías AA de 900mAh por debajo del límite de operación del módulo XBee $(2.2 v)$. Este procedimiento es empleado para comparar el consumo de energía en otros protocolos de comunicaciones.

Figura 10. Consumo de energía real y simulada

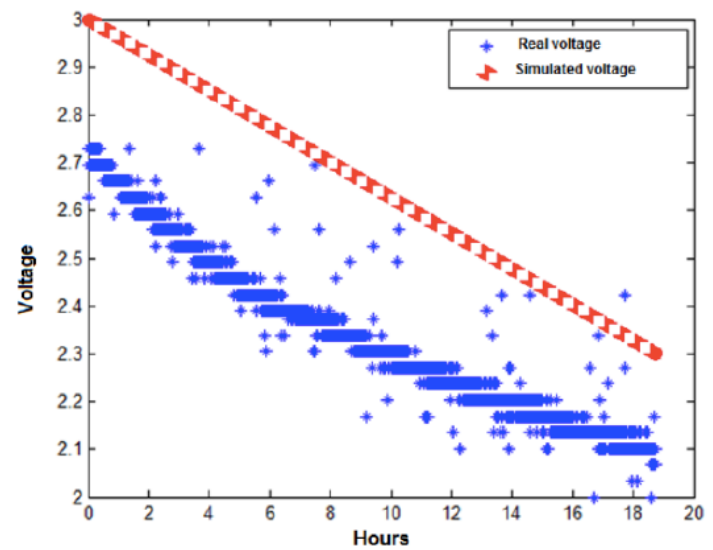

\section{IMPLEMENTACIÓN EN ALGORITMOS DE ENRUTAMIENTO}

Adhoc On-demand Distance Vector (AODV) Perking, C. y Royer E, 2000 es un algoritmo de enrutamiento de referencia por excelencia, ya que es distribuído, bajo demanda y sencillo 


\section{Año 13.}

Revista de Investigación

Núm. 33

Académica sin Frontera

ISSN: 2007-8870

\section{http://revistainvestigacionacademicasinfrontera.com}

Recibido el 15 de octubre de 2020. Dictaminado mediante arbitraje favorablemente 20 de diciembre de 2020.

de implementar. Está basado en el intercambio de paquetes de señalización, descubrimiento de vecinos y de rutas hacia pares fuente destino.

El descubrimiento de rutas en AODV inicia con una inundación intencional de un paquete de petición de ruta (Route Request - RREQ). Eventualmente este paquete alcanza su destino, el cual responde utilizando la trayectoria en reversa mediante un paquete de respuesta de ruta (Route Response - RREP). Cuando este paquete alcanza el nodo fuente, se inicia la transmisión de datos por la ruta descubierta. Esta ruta tiene un tiempo de vida, por lo que debe mantenerse activa para evitar requerir descubrir de nuevo una ruta.

Figura 11. Mensaje de control AODV

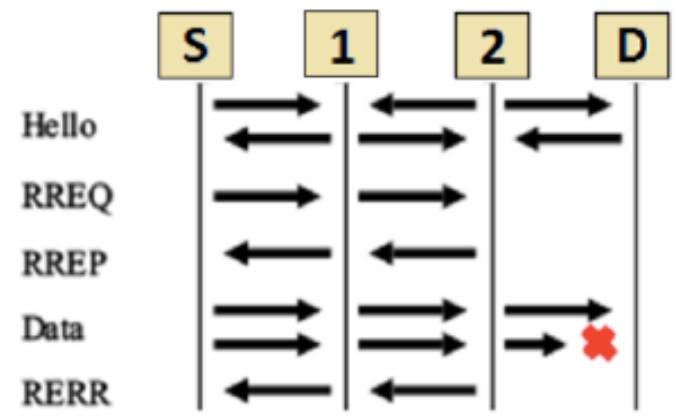

La Figura 11 muestra el intercambio de paquetes en AODV para establecer comunicación entre una fuente (S) y un destinatario (D) a través de los nodos 1 y 2.

QoS capable cross-layer mac protocol for wireless sensor networks (QUATTRO) Ruiz, et. al. 2009 es un protocolo de coordinación para WSN centralizado, proactivo, con asignación de pesos, reservación de rutas, estimación de interferentes y ventanas de actividad calendarizadas. QUATTRO ofrece garantías de QoS y bajos consumos de energía; está basado en una fase de configuración donde cada nodo anuncia el tráfico que debe soportar a 
Año 13.

Revista de Investigación

Núm. 33

\section{http://revistainvestigacionacademicasinfrontera.com}

Recibido el 15 de octubre de 2020. Dictaminado mediante arbitraje favorablemente 20 de diciembre de 2020.

priori, para que el Sink calcule ventanas de actividad reservadas para cada nodo de la red. QUATTRO considera seis fases:

1.- Descubrimiento de rutas y asignación de pesos.

2.- Selección y reservación de rutas.

3.- Estimación de interferencias.

4.- Calendarización de agrupamientos con ventanas de actividad para recepción de datos.

5.- Fase de transmisión de datos.

6.- Procesos de detección de fallas de enlaces y reconfiguración de rutas.

QUATTRO basa su capacidad de proveer QoS en una asignación ponderada de reservación de rutas. La probabilidad de escoger una posible ruta hacia el Sink está dada por la ecuación 2 (Ruiz et. al. 2009).

$$
p_{i}=\frac{\varepsilon_{i}}{\lambda_{i}\left(h_{i}\right)^{\beta}}, \quad, \text { para } \mathrm{i}=1,2, \ldots, \mathrm{N}
$$

\section{Donde:}

$\varepsilon_{i}=$ Cuello de botella de la $i$-ésima ruta

$\chi=$ Carga en el cuello de botella de la i-ésima ruta

$h_{i}=$ Número de saltos en la i-ésima ruta

$\beta=(0,1)$ factor de impacto del número de saltos

La ecuación (2) le da equidad a la selección de rutas, permite un bajo consumo de canales en un esquema de acceso.múltiple, mejorando la carga de tráfico orientándolas a rutas más 
Año 13.

Revista de Investigación

Académica sin Frontera

Núm. 33

ISSN: 2007-8870

\section{http://revistainvestigacionacademicasinfrontera.com}

Recibido el 15 de octubre de 2020. Dictaminado mediante arbitraje favorablemente 20 de diciembre de 2020. aptas. La ecuación 2 le da mayor probabilidad de ser elegida a una ruta con mayor reserva de energía y menor carga de tráfico sin descartar completamente aquellas rutas más congestionadas y restringidas.

La Figura 12 muestra un escenario con una densidad de 20 nodos por área de cobertura, distribuidos aleatoriamente en un espacio de 400x400m. Tienen un rango de transmisión típico de un nodo XBee S1 de 100m. El nodo 64 es el Sink.

Figura 12. Escenario con una densidad de 20n/Ac

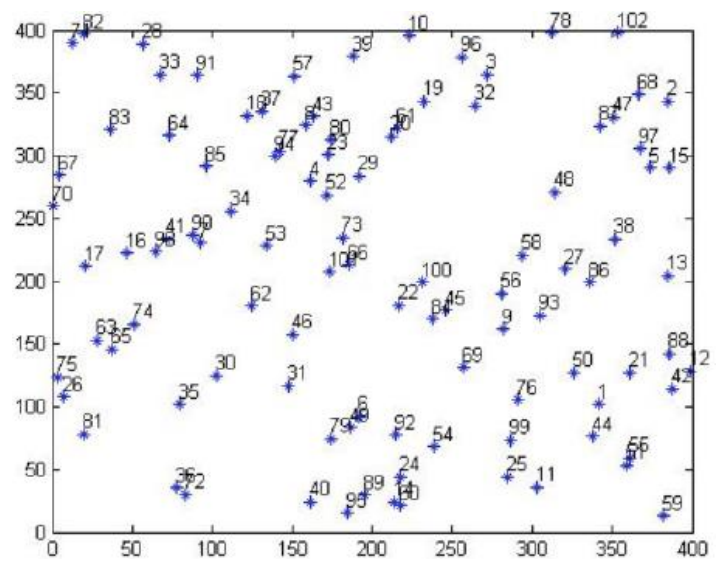

La Figura 13 muestra el tiempo de configuración del algoritmo AODV y QUATTRO durante el proceso de descubrimiento de rutas. Los mensajes de inundación en AODV producen mayores retrasos que los mensajes de configuración en QUATTRO. 
"El saber de mis hijos

Año 13.

Núm. 33
Revista de Investigación

Académica sin Frontera

ISSN: 2007-8870

\section{http://revistainvestigacionacademicasinfrontera.com}

Recibido el 15 de octubre de 2020. Dictaminado mediante arbitraje favorablemente 20 de diciembre de 2020.

Figura 13. Tiempo de configuración en segundos AODV y QUATTRO

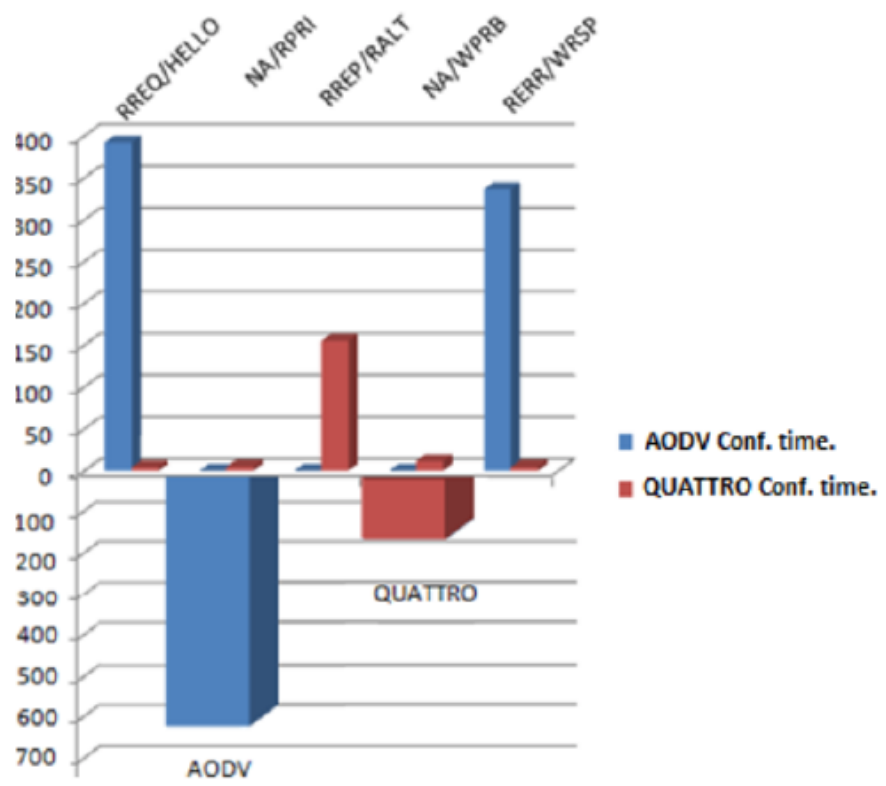

La Figura 14 muestra el consumo de energía acumulado en AODV y QUATTRO. Aun cuando ambos protocolos producen una inundación en la red, QUATTRO consume menor energía que AODV. QUATTRO está diseñado para enrutar paquetes hacia un Sink, lo que produce que la inundación sea controlada y más eficiente.

Figura 14. Energía consumida (Joule), fase de configuración

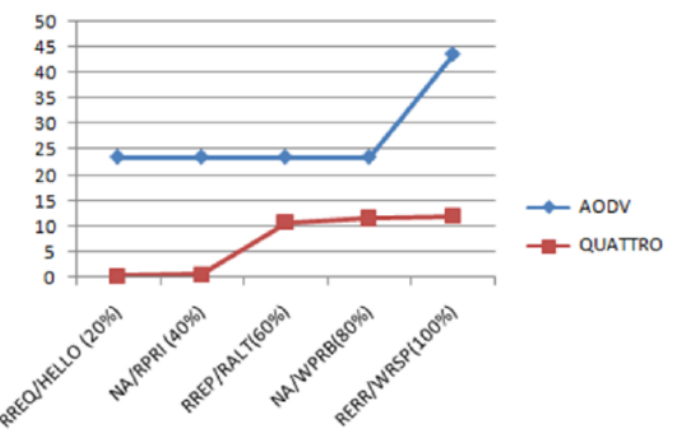




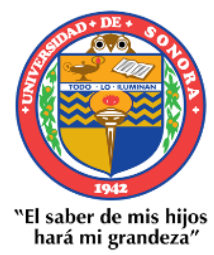

Año 13.

Núm. 33
( Julio - Diciembre 2020)

Revista de Investigación Académica sin Frontera ISSN: 2007-8870

\section{http://revistainvestigacionacademicasinfrontera.com}

Recibido el 15 de octubre de 2020. Dictaminado mediante arbitraje favorablemente 20 de diciembre de 2020.

\section{CONCLUSIONES Y TRABAJO FUTURO}

En este trabajo, se ha presentado una API con distintas funciones que ofrece calidad y robustez para el desarrollo de aplicaciones orientadas al IoT. También se desarrolló un sensor para realizar pruebas de consumo de energía en un entorno real.

Para probar el consumo de energía utilizando la API y el sensor, se evaluaron los protocolos AODV y QUATTRO en entorno de simulación y en entorno real, demostrando que el protocolo de enrutamiento QUATTRO produce menos mensajes en la fase de descubrimiento, y por consiguiente, el consumo de energía de QUATTRO es menor que el de AODV. Los resultados son similares tanto en la simulación como en el entorno real.

La API desarrollada permite una rápida integración con aplicaciones orientadas al IoT, y se encuentra en la fase de evaluación en los desarrollos: a) transmisión inalámbrica de señales de ultrasonido para evaluación no destructiva de estructuras y b) transmisión de datos de un medidor de gas L.P. 
http://revistainvestigacionacademicasinfrontera.com

Recibido el 15 de octubre de 2020. Dictaminado mediante arbitraje favorablemente 20 de diciembre de 2020.

\section{REFERENCIAS}

- Anastasi, G., Conti, M., Francesco, M., Passarella, A., Energy conservation in wireless sensor networks: a survey, Ad Hoc Networks 7(3), pp. 537-568 (2009).

- Chen, S., Xu, H., Liu, D., Hu, B., Wang, H., A vision of IoT: Applications, Challenges, and Opportunities with China Perspective. IEEE Internet of Things Journal, 1(4), 349-359 (2014).

- Dengler, S., Awad, A.,Dressler, F., Sensor/Actuator Networks in Smart homes for Supporting Elderly and handicapped People, Interantional Conference on Advanced Information Networking and Applications Workshops, pp. 863-868, 2007.

- Fall, K., Varadhan, K., ns Notes and Documents, The VINT Project. UC Berkeley, LBL, USC/ISI, and Xerox PARC, 2000.

- Ferronato, J.J. y Trentin, M.A.S., Analysis of Routing Protocols OLSR, AODV and ZRP in Real Urban Vehicular Scenario with Density Variation. IEEE Latin America Transactions 15(9), pp. 1727-1734 (2017).

- From the Death Criterion Perspective. International Journal of Handheld Computing Research, DOI: 10.4018/IJHCR.2017070103. (2017).

- Fotino, M., Gozzi, A., Cano, J.C., Calafate, C., De Rango, F., Manzoni, P., Marano, S., Evaluating energy consumption of proactive and reactive routing protocols in a MANET. IFIP International Federation for Information Processing., Vol. 248, Wireless Sensor and Actor Networks, pp. 119-130 (2007).

- Hung, M., Leading the IoT - Gartner Insights on How to Lead in a Connected World. Gartner, 2017.

- Giannoulis, S., Antonopoulos, C., Topalis, E., Koubias, S., ZRP versus DSR and TORA: A comprehensive survey on ZRP performance, IEEE Transactions on Industrial Informatics 3(1), 63-72 (2007).

- Jiang, M., Li, J., Tay, Y.C., Cluster Based Routing Protocol (CBRP), draft-ietfmantcbrp-spec-01.txt, 1999.

- Johnson, D., Maltz, D.A. Dynamic Source Routing in Ad Hoc Wireless Networks. Mobile Computing, pp.153-181 (1996).

- Kanakaris, V., Ndzi, D., Azzi, D., Ad-hoc Networks energy consumption: A review of the ad-hoc routing protocols. Journal of Engineering and Technology Review 3(1) pp. 162-167 (2010). 


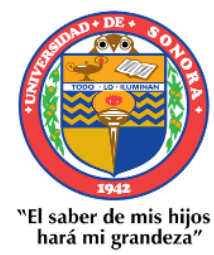

( Julio - Diciembre 2020)

Año 13.

Núm. 33

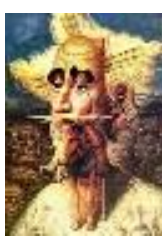

Revista de Investigación Académica sin Frontera

ISSN: 2007-8870

http://revistainvestigacionacademicasinfrontera.com

Recibido el 15 de octubre de 2020. Dictaminado mediante arbitraje favorablemente 20 de diciembre de 2020.

- Nadilma, C.V.N Pereira y Renato M. de Moraes, Comparative analysis of AODV route recovery mechanisms in wireless ad hoc networks. IEEE Latina America Transactions 8(4), pp. 385-393 (2010).

- Paul R. Santiago N. Stalin R. Vanessa A. Luis B. Christian R. Internet de las cosas y redes de sensors inalámbricos: Review. Sistemas embebidos: Estado actual con vision al futuro. (2017).

- Pascual Espada, J., Diseño de objetos virtuales colaborativos orientados a servicios en el marco de Internet de las cosas. Universidad de Oviedo, 1-279 (2012).

- Perking, C., Royer, E., Ad-hoc On-Demand Distance Vector (AODV) Routing, Internet Draft, MANET working group, draft.ietf-manet-aodv-05.txt, 2000.

- Ruiz, J. Garlladro, J.R., Villasenor-Gonzlaez, L., Makrakis, D., Mouftah, H.T., QUATTRO: QoS capabale cross-Layer MAC protocol for wireless sensor networks, Global Telecommunications Conference, GLOBECOM, PP. 1-6, (2009).

- Vermesan, O. Friess, P., Internet of Things - from research and innovation to market deployment. River Publishers, Dinamarca, 2014.

- Sara N. Nada E. Ahmed K. Samy S. Ramez M. Hassanein H. WSN Lifetime and Reliability Analysis

- Srivastava, A., Mishra, A., Upadhyay, S., A conceptual overview of energy consumption based routing protocol - ECBR. International Journal of Computer Applications 92(3), 18-22 (2014).

- Sultan, T. M., Zaki, S. M., Evaluation of energy consumption of reactive and proactive routing protocols in MANET. International Journal of Computer Networks \& Communications 9(2), pp. 29-38 (2017).

- W. Hu, H.H. Li, W.H. Yao, Y.W. Hu, Energy Optimization for WSN in Ubiquitous Power Internet of Things. INTERNATIONAL JOURNAL OF COMPUTERS COMMUNICATIONS \& CONTROL, Shangai, China (2019).

- Zhang, Y., Hsiao-Wa, C., Mohsen, G., Cooperative Wireless Communciations, 2009.

- Zorzi, M., Gluhak, A.A., Lange, S., Bassi, A., From today's INTRAnet of Things to a Future INTERnet of Things: A Wireless- and Mobility- Related View. IEEE Wireless Communications, 17(6), 44-51 (2010). 
"El saber de mis hijos

Año 13.

Núm. 33
Revista de Investigación

Académica sin Frontera

ISSN: 2007-8870

http://revistainvestigacionacademicasinfrontera.com

Recibido el 15 de octubre de 2020. Dictaminado mediante arbitraje favorablemente 20 de diciembre de 2020.

\section{Directorio Institucional}

Dr. Enrique Fernando Velázquez Contreras

Rector

Dr. Ramón Enrique Robles Zepeda

Secretario General Académico

Dra. Rosa María Montesinos Cisneros

Secretaria General Administrativa

Dr. Rodolfo Basurto Álvarez

Director de Vinculación y Difusión

Dra. Adriana Leticia Navarro Verdugo

Vicerrectora de la Unidad Regional Sur

Dr. Ernesto Clark Valenzuela

Director de la División de Ciencias Económicas y Sociales

Dr. Francisco Espinoza Morales

Secretario de la División de Ciencias Económico y Sociales

Dra. Leticia María González Velásquez

Jefe del Departamento de Ciencias Económico Administrativas

Dra. Lidia Amalia Zallas Esquer

Jefe de Departamento de Ciencias Sociales 
Año 13.

Revista de Investigación

Núm. 33

Académica sin Frontera

ISSN: 2007-8870

http://revistainvestigacionacademicasinfrontera.com

Recibido el 15 de octubre de 2020. Dictaminado mediante arbitraje favorablemente 20 de diciembre de 2020.

\title{
Comité Directivo
}

\author{
Editor Responsable \\ Dr. Francisco Espinoza Morales \\ Universidad de Sonora \\ Directora \\ Dra. Leticia María González Velásquez \\ Universidad de Sonora \\ Subdirector \\ Dr. Javier Carreón Guillen \\ Universidad Nacional Autónoma de México \\ Editor Científico \\ Dr. Cruz García Lirios \\ Universidad Autónoma del estado de México \\ Master Gráfico \\ M.T.I. Francisco Alan Espinoza Zallas \\ Universidad Estatal de Sonora
}

Nos complace anunciar que su diario, "Academic Research Journal Withoutborders" (ISSN/EISSN

2007-8870) fue evaluado positivamente en la indexación Citefactor, ahora la página de la revista está disponible en línea, en caso de cualquier problema.

Journals Master | International Innovative Journal Impact Factor (IIJIF)

Red Latinoamericana de revistas Académicas en Ciencias Sociales y Humanidades
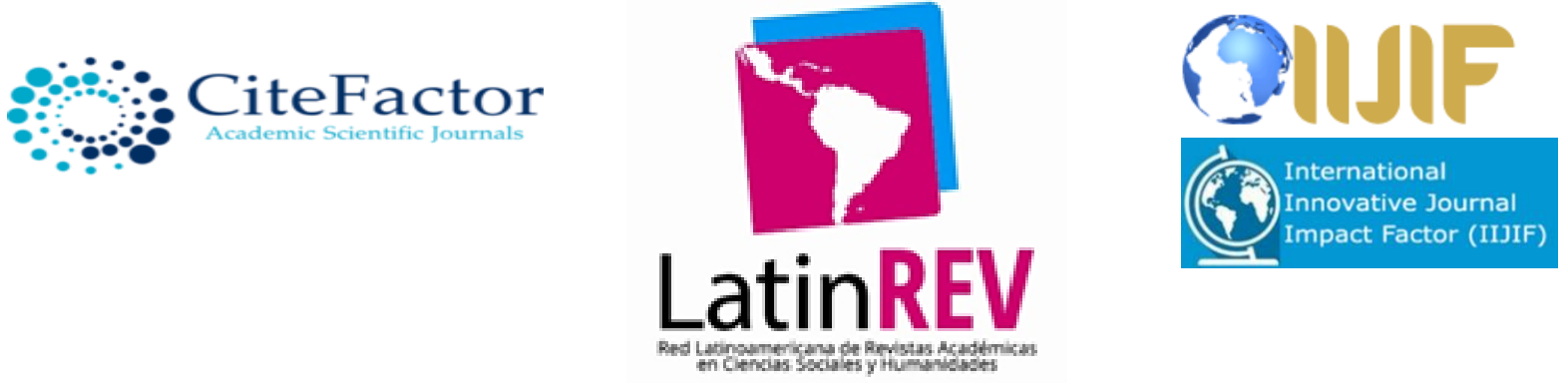
"El saber de mis hijos

Año 13.

Núm. 33
Revista de Investigación

Académica sin Frontera

ISSN: 2007-8870

http://revistainvestigacionacademicasinfrontera.com

Recibido el 15 de octubre de 2020. Dictaminado mediante arbitraje favorablemente 20 de diciembre de 2020.

\section{Comité editorial}

Dra. Angélica María Rascón Larios

Universidad de Sonora. México

Dra. María del Rosario Molina González

Universidad de Sonora

Dra. Francisca Elena Rochin Wong

Universidad de Sonora. México

Dra. Lidia Amalia Zallas Esquer

Universidad de Sonora. México

Dra. Beatriz Llamas Arechiga

Universidad de Sonora. México

Dr. Rogelio Barba Álvarez

Universidad de Guadalajara. México

Dra. Rosa María Rincón Ornelas

Universidad de Sonora. México

Dr. Juan Flores Preciado

Universidad de Colima. México

Dr. Amado Olivares Leal. Universidad de Sonora

Universidad de Sonora. México

Dr. Guillermo Velázquez Valadez.

Instituto Politécnico Nacional (IPN) México

Dr. Hugo Nefstalí Padilla Torres.

Universidad Estatal de Sonora. México

Dr. Luis Ramón Moreno Moreno.

Universidad Autónoma de Baja California. México 


\section{Año 13.}

Revista de Investigación

Núm. 33

ISSN: 2007-8870

\section{http://revistainvestigacionacademicasinfrontera.com}

Recibido el 15 de octubre de 2020. Dictaminado mediante arbitraje favorablemente 20 de diciembre de 2020.

Dr. Miguel Ángel Vázquez Ruiz.

Universidad de Sonora. México

Dra. Lorena Vélez García.

Universidad Autónoma de Baja California. México

Dra. Pabla Peralta Miranda.

Universidad Simón Bolívar, Barranquilla, Colombia

Mtro. Roberto Espíritu Olmos

Universidad de Colima (FCA Tecomán) Colima

Dr. Héctor Priego Huertas.

Universidad de Colima (FCA Tecomán) Colima

Mtra. María Guadalupe Alvarado Ibarra.

Universidad de Sonora. México.

MSc. Celso Germán Sánchez Zayas

Universidad de Camagüey, Ignacio Agramonte Loynaz, Cuba

Dra. María Luisa Quintero Soto

Universidad Autónoma del Estado de México

Dr. Eyder Bolivar Mojica

Universidad Católica, Luis Amigó, Medellin, Colombia

Revisores de Textos en Inglés

Mtro. Renato Encinas

Mtra. Cecilia Guadalupe Martínez Solano

\section{Comité científico}

Dr. Rosendo Martínez Jiménez. Universidad Autónoma Benito Juárez de Oaxaca.

Dr. Hugo Neftalí Padilla. Universidad Estatal de Sonora

Dra. María Teresa Gaxiola Sánchez. Universidad de Sonora.

Dr. José Cesar Kaplan. Universidad Estatal de Sonora. 


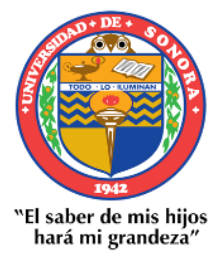

( Julio - Diciembre 2020)

Año 13.

Revista de Investigación

Núm. 33

Académica sin Frontera

ISSN: 2007-8870

http://revistainvestigacionacademicasinfrontera.com

Recibido el 15 de octubre de 2020. Dictaminado mediante arbitraje favorablemente 20 de diciembre de 2020.

Dr. Alfredo Islas Rodríguez. Universidad de Sonora

Frecuencia de publicación: semestral / 2 números por año.

Revista de Investigación Académica sin Frontera (RIASF) con (ISSN: 2007-8870) es un interlocutor internacional de acceso abierto revisado diario en línea en el ámbito del de las Ciencias Económicas Administrativas y Sociales. Su objetivo principal es dar a los trabajos de investigación de calidad. Cubre todas las sub-campos de los campos anteriormente mencionados. Proporciona la plataforma a académicos, estudiantes y profesionales. Sólo pública trabajos de investigación y artículos de revisión inicial. Documento presentado debe cumplir con algunos criterios como, debe ser original, inédita y no estén sometidos a ninguna otra revista.

RIASF es una revista arbitrada / Revisión por pares International. Publicamos documentos sobre una variedad de temas, contextos y estrategias de análisis que examinan la relación entre la rápida evolución para la Sociedad y la tecnología del conocimiento.

REVISTA DE INVESTIGACIÓN ACADÉMICA SIN FRONTERA, Año 13, No. 33, Julio - diciembre 2020, es una publicación semestral de investigación científica, editada por la Universidad de Sonora, a través de las División de Ciencias Económicas y Sociales, de la Unidad Regional Sur, Blvd. Lázaro Cárdenas No. 100, Col. Francisco Villa, Navojoa, Sonora, Sonora, México, C.P. 85880. Tel. (642) 42599-54.

http://www.revistainvestigacionacademicasinfrontera.com/, revistaacademicasinfrontera@unison.mx.

Editor responsable: Francisco Espinoza Morales. Reserva de Derechos al Uso Exclusivo: 042013-121811323700-203 e ISSN: 2007-8870, ambos otorgados por el Instituto Nacional de Derecho de Autor. Inscrita en el Directorio de LATINDEX, con Núm. De folio 20014, folio único 14590. Responsable de la última actualización de este Número, Unidad Informática de la Universidad de Sonora, fecha de la última modificación, 30 de diciembre 2020, indexada a Cite Factor Academic Scientific Journal y Journals Master (IIJIF) y Red Latinoamericana de Revistas Académicas en Ciencias Sociales y Humanidades, (Latín Rev). Las opiniones expresadas por los autores no necesariamente reflejan la postura del editor de la publicación. Se autoriza la reproducción total o parcial de los contenidos e imágenes en la presente publicación siempre y cuando se cuente con la autorización del editor y se cite plenamente la fuente. 
Año 13.

Revista de Investigación

Académica sin Frontera

Núm. 33

ISSN: 2007-8870

\section{http://revistainvestigacionacademicasinfrontera.com}

Recibido el 15 de octubre de 2020. Dictaminado mediante arbitraje favorablemente 20 de diciembre de 2020.

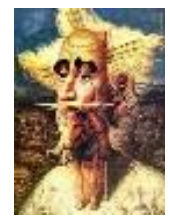

Nos complace anunciar que su diario, "Academic Research Journal Withoutborders" (ISSN/EISSN 2007-8870) fue evaluado positivamente en la indexación Citefactor, ahora la página de la revista está disponible en línea, en caso de cualquier problema.

Journals Master | International Innovative Journal Impact Factor (IIJIF)

Red Latinoamericana de revistas Académicas en Ciencias Sociales y Humanidades

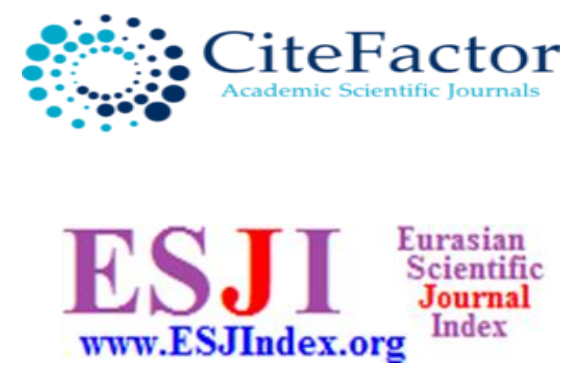

https://www.neliti.com

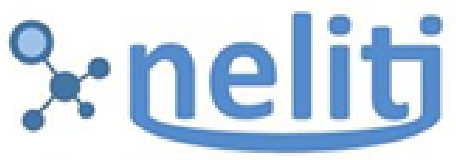

Indonesia's Research Repository
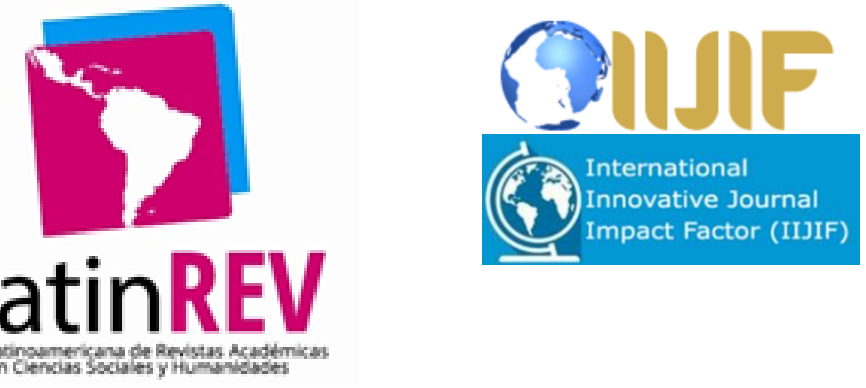
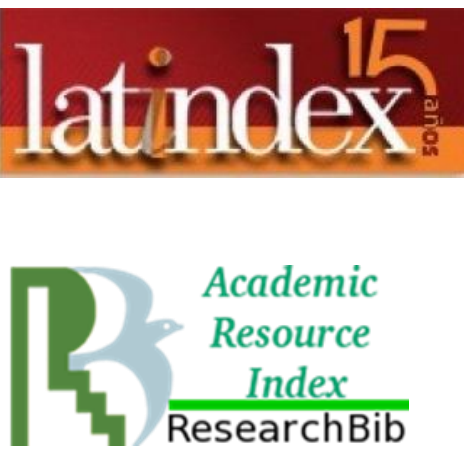